\title{
Operando Measurement of Layer Breathing Modes in Lithiated Graphite
}

\author{
Hossein Yadegari, ${ }^{*+}$ Mohamed A. Koronfel, ${ }^{+,}{ }^{\ddagger}$ Kang Wang, ${ }^{+}$Daisy B. Thornton, ${ }^{+}$Ifan E.L. \\ Stephens, ${ }^{+}$Carla Molteni, ${ }^{z}$ Peter D. Haynes, ${ }^{+}$Mary P. Ryan ${ }^{*}+$ \\ ${ }^{\dagger}$ Department of Materials, Imperial College London, London SW7 2AZ, U.K. \\ ${ }^{\ddagger}$ Diamond Light Source, Harwell Science and Innovation Campus, Didcot OX11 ODE, U.K. \\ Department of Physics, King's College London, Strand, London WC2R 2LS, U.K.
}

*Prof. Mary P. Ryan, E-mail: m.p.ryan@imperial.ac.uk, Dr. Hossein Yadegari, E-mail: h.yadegari@imperial.ac.uk 


\section{'Experimental}

\section{Operando Raman Spectroscopy}

Operando Raman Spectroscopy measurements were performed in a bespoke stainless-steel Raman cell equipped with a glass window $(0.5 \mathrm{~mm}$ thick) as shown in Figure S1. Graphite electrodes were prepared by casting a mixture of natural graphite powder (WMG, UK) and polyvinylidene fluoride (PVDF, Alfa Aesar) with a weight ratio of 9:1 on a separator (Celgard 2325). The electrodes were $3 / 8$ inch in diameter with a loading capacity of $\sim 0.25 \mathrm{mg}$ (equivalent to an areal loading of $0.35 \mathrm{mg} \mathrm{cm}^{-2}$ ). A low loading was selected to minimize the mass transfer resistance through the electrode and ensure a uniform state of charge (SoC), as the Raman measurements were conducted on the outer electrode surface. A copper mesh was placed between the glass window and graphite electrode as the current collector. Lithium foil on a stainless-steel current collector and glass fiber (GF/A, Sigma Aldrich) were used as the negative electrode and separator, respectively. Electrolyte was $1 \mathrm{M} \mathrm{LiPF}_{6}$ in EC/EMC with 3/7 weight ratio provided by SoulBrain. Raman spectra were collected using an inVia Renishaw confocal Raman microscope operated with an incident laser beam at $532 \mathrm{~nm}$ focused through a 50x objective (Leica). The laser intensity was set to $<1 \mathrm{~mW}$ and Raman spectra were collected under static mode with the exposure time of $1 \mathrm{~s}$ in 5 -minute intervals to minimize the sample heating. The laser shutter was kept closed between spectra collections.

\section{Density Functional Theory (DFT) Calculations}

The simulation results are obtained from density-functional theory (DFT), using the CASTEP 18.1 package $^{1}$ with PBE exchange and correlation functional. ${ }^{2}$ To account for the van der Waals interactions between the carbon layers, we include a dispersion correction to the total energy of the system using the Grimme scheme ${ }^{3}$ as implemented in CASTEP. ${ }^{4}$ With the van der Waals correction, the ionic positions are fully relaxed and then the relaxed structure is used to calculate the phonon dispersion. All the calculations are performed with norm-conserving 
pseudopotentials with a cutoff energy of $1050 \mathrm{eV}$. The structure relaxation is performed using the Broyden-Fletcher-Goldfarb-Shanno (BFGS) algorithm ${ }^{5}$ with force tolerance of $0.01 \mathrm{eV} \AA^{-1}$, stress tolerance of $0.01 \mathrm{GPa}$ and a $\Gamma$-centered Monkhorst-Pack k-point grid $11 \times 11 \times 5$ for $\mathrm{Li}_{2} \mathrm{C}_{12}$, $\mathrm{LiC}_{12}$ and $\mathrm{LiC}_{18}$. Phonon dispersion is performed using the finite displacement / supercell method. The unit cells for phonon dispersion is chosen to be $\mathrm{Li}_{2} \mathrm{C}_{12}, \mathrm{LiC}_{12}$ and $\mathrm{LiC}_{18}$. The low frequency Raman active mode is shown in the phonon dispersion figures (Figure 3 and S12-14) with red color. 


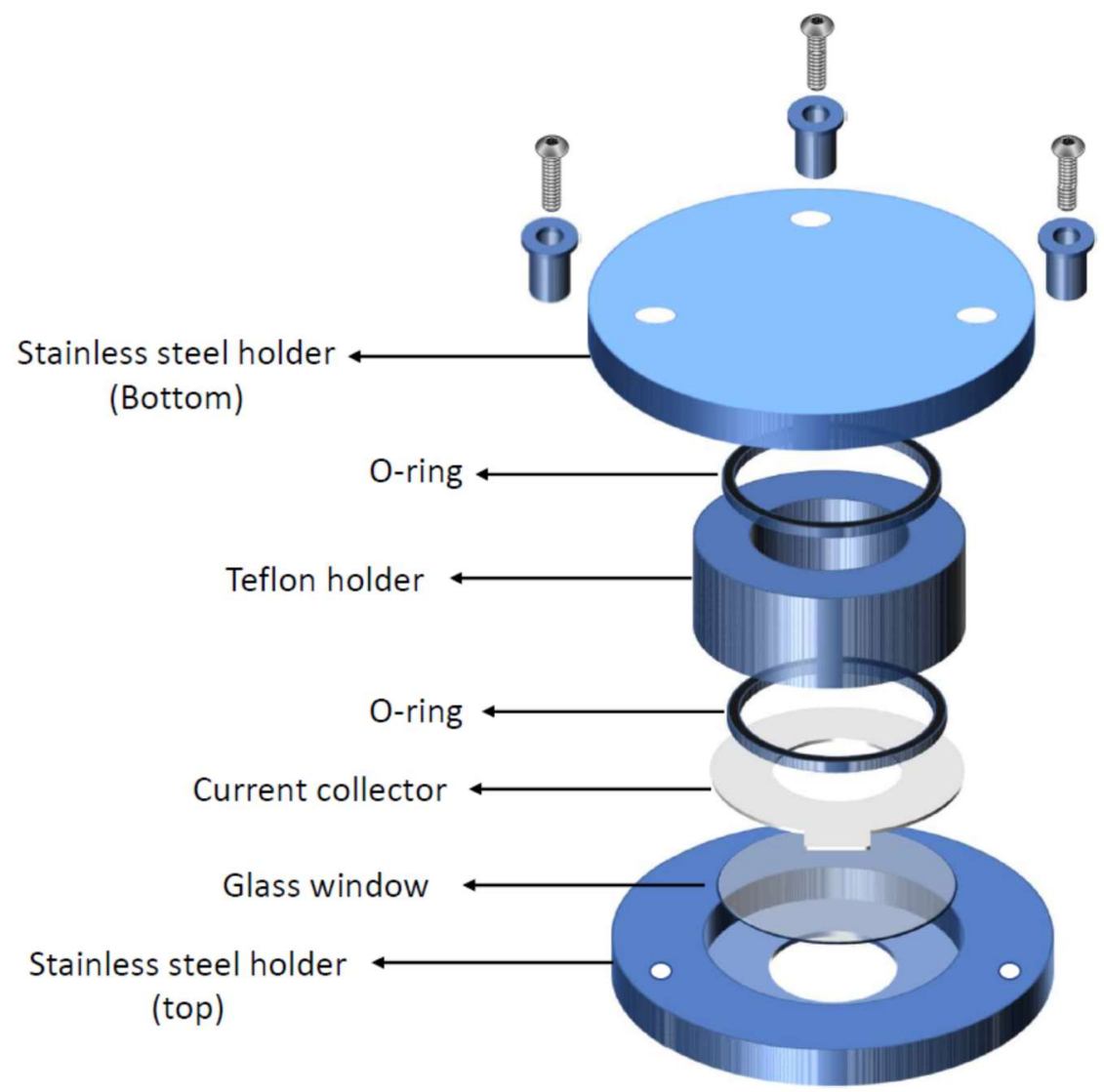

Figure S1: A schematic of the operando Raman cell assembly. A bespoke operando Raman cell with a glass window was designed and fabricated in this study. Graphite electrode was directly casted on Celgard separator and used as the positive electrode against Li metal in a half-cell configuration. 


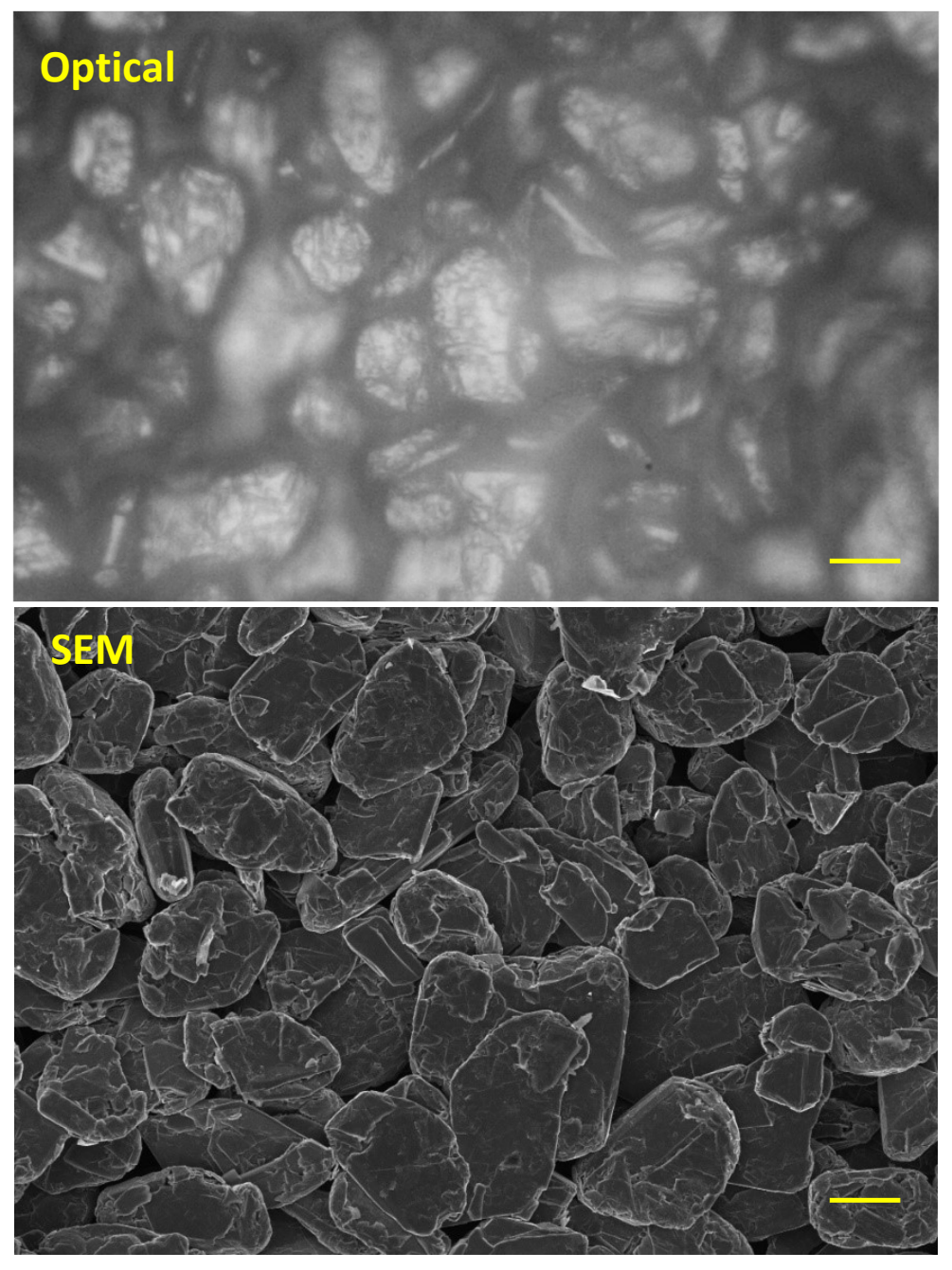

Figure S2: The SEM image of the as-prepared graphite electrode is presented alongside the optical image obtained from the Raman microscope on the assembled half-cell. The optical image obtained from the Raman microscope using a 50x objective on the assembled half-cell (top); SEM image of the as-prepared graphite electrode (bottom). The scale bars represent $10 \mu \mathrm{m}$. 


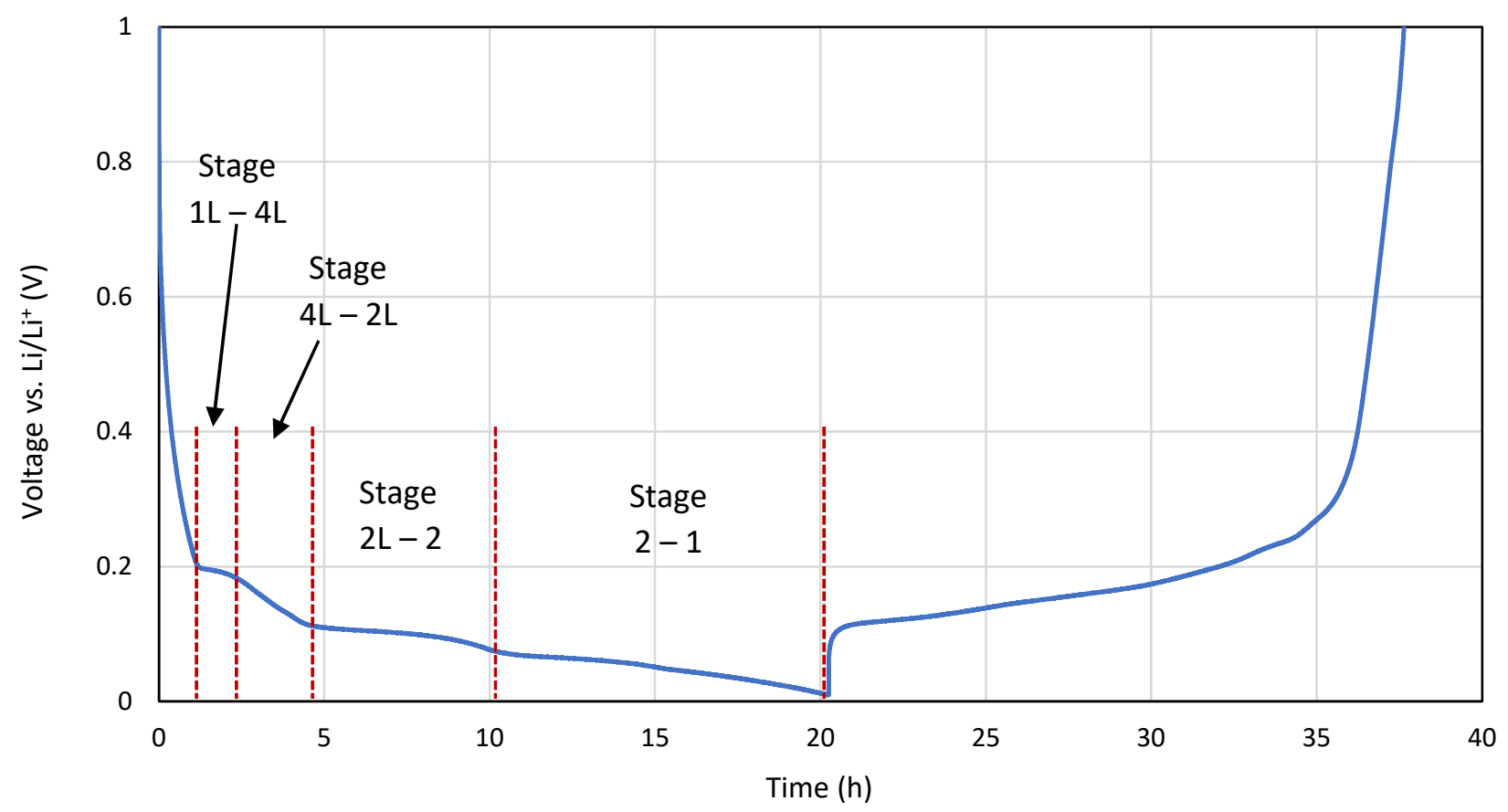

Figure S3: A typical galvanostatic charge/discharge profile of the half-cell cycled between 1.0 and $0.01 \mathrm{~V}$ vs. $\mathrm{Li}^{\mathrm{Li}}{ }^{+}$in the operando Raman cell at $\mathrm{C} / 20$. 


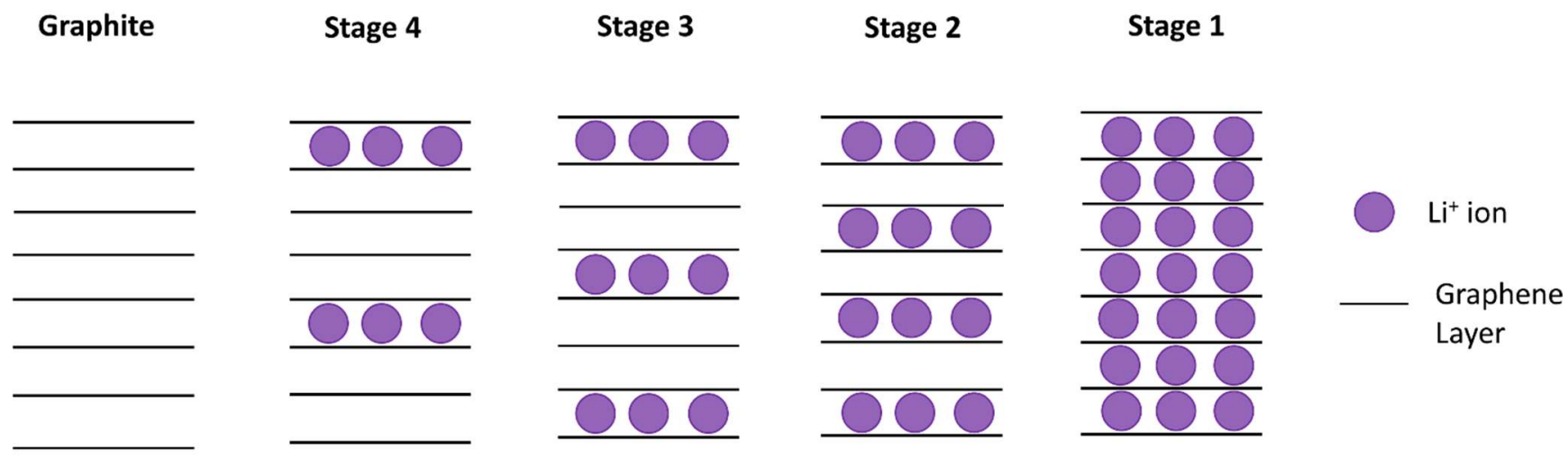

Figure S4: Intercalation of $\mathrm{Li}^{+}$into the graphite is described by a stage index, $n$, representing the number of graphene layers between two guest layers. 


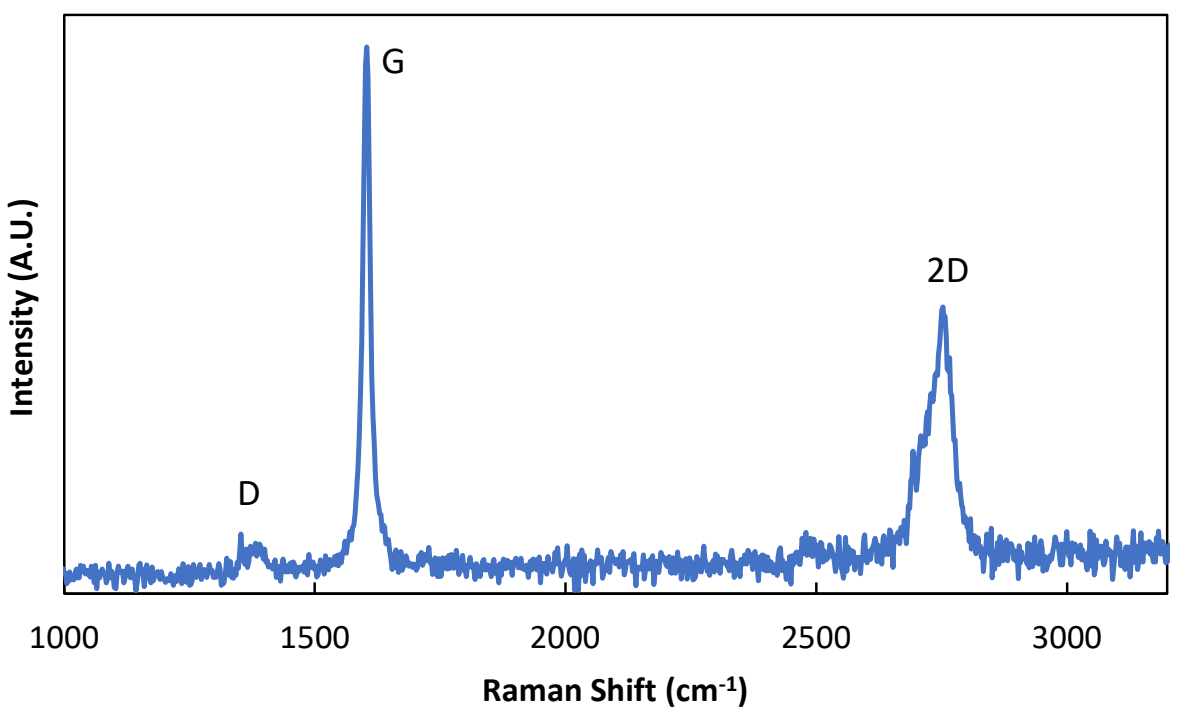

Figure S5: A typical Raman spectrum recorded using a fresh graphite electrode. 

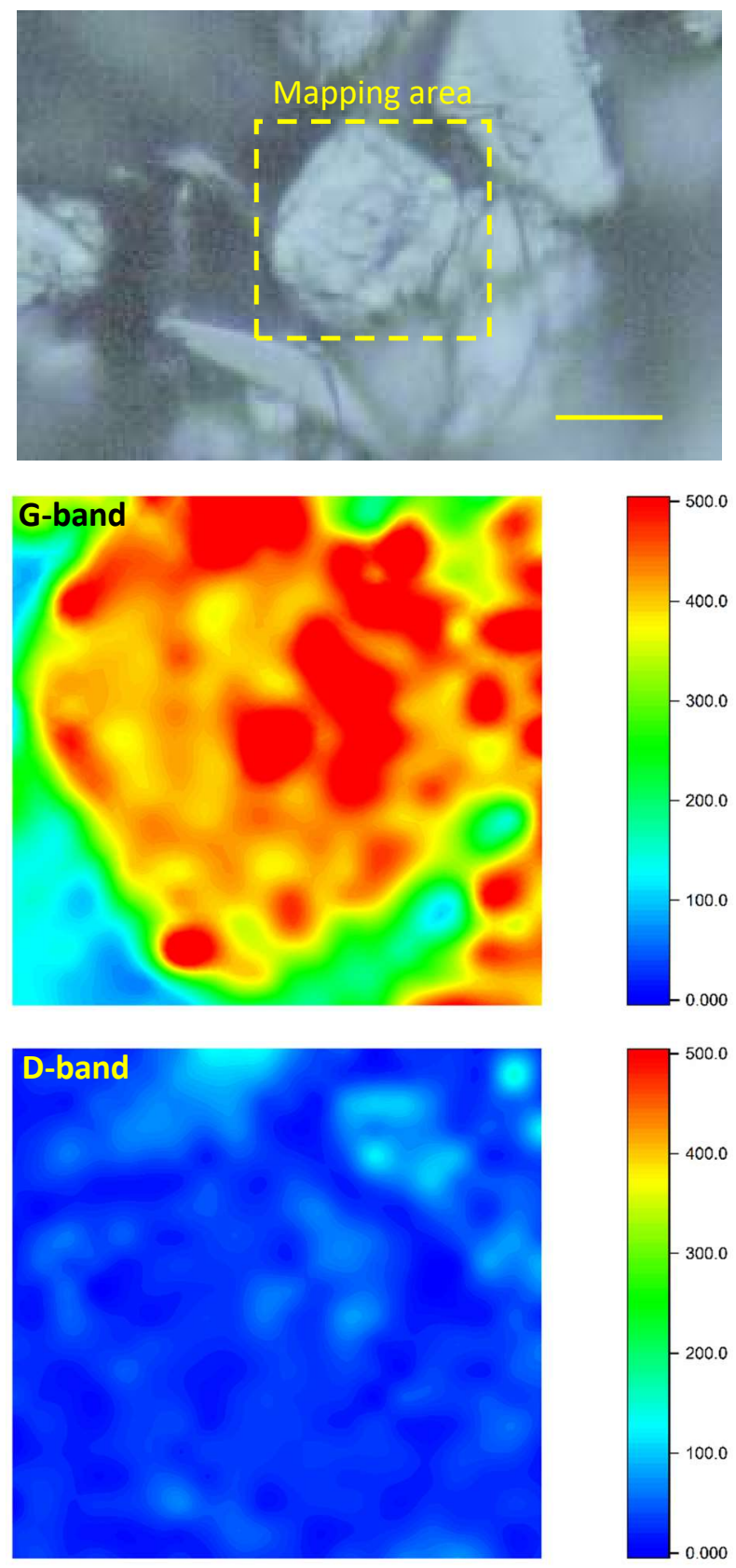

Figure S6: Typical chemical maps of $D$ and $G$ bands of a single graphite particle recorded on the specified area on the optical image. The scale bar on the optical image represents $10 \mu \mathrm{m}$. The chemical maps demonstrate that the electrode is mainly composed of crystalline graphite with traces of disordered carbon. 


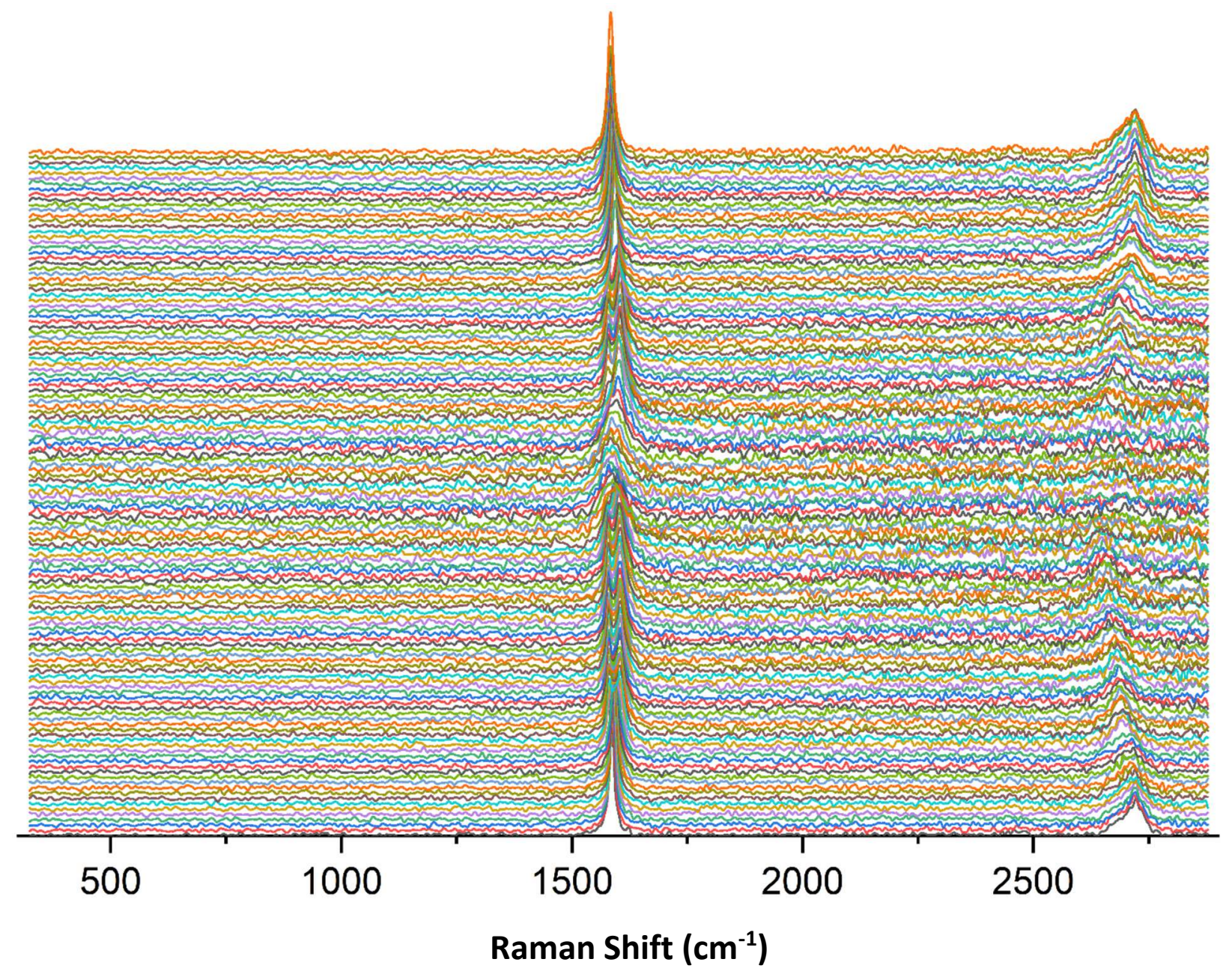

Figure S7: Operando Raman spectra in a wide range of frequencies obtained from a typical charge/discharge cycle of graphite electrode in a half-cell vs. Li electrode performed under C/2. $G$ and $2 D$ bands were the only active features in the range of 300 to $2900 \mathrm{~cm}^{-1}$. No Raman active peak related to the other components of the cell including binder and electrolyte was detected, ruling out the potential interference with the graphite response. 


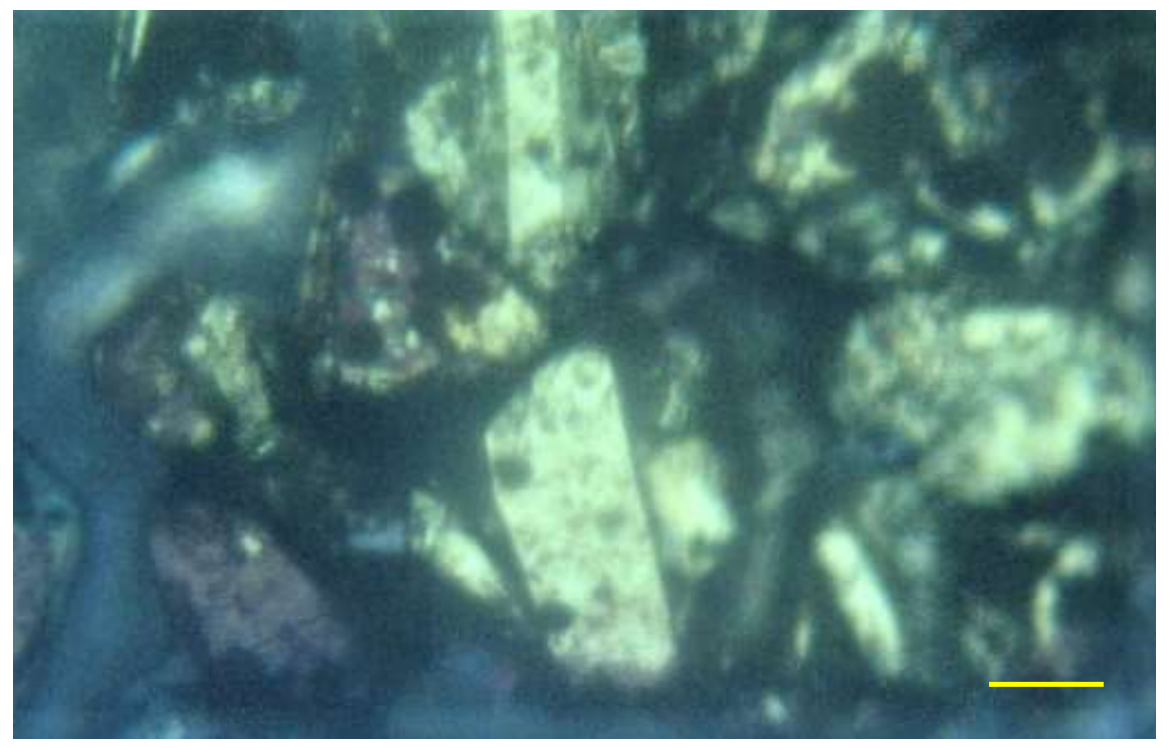

Figure S8: An optical image of intercalated graphite electrode obtained from Raman microscope using 50x objective. The scale bar represents $10 \mu \mathrm{m}$. 


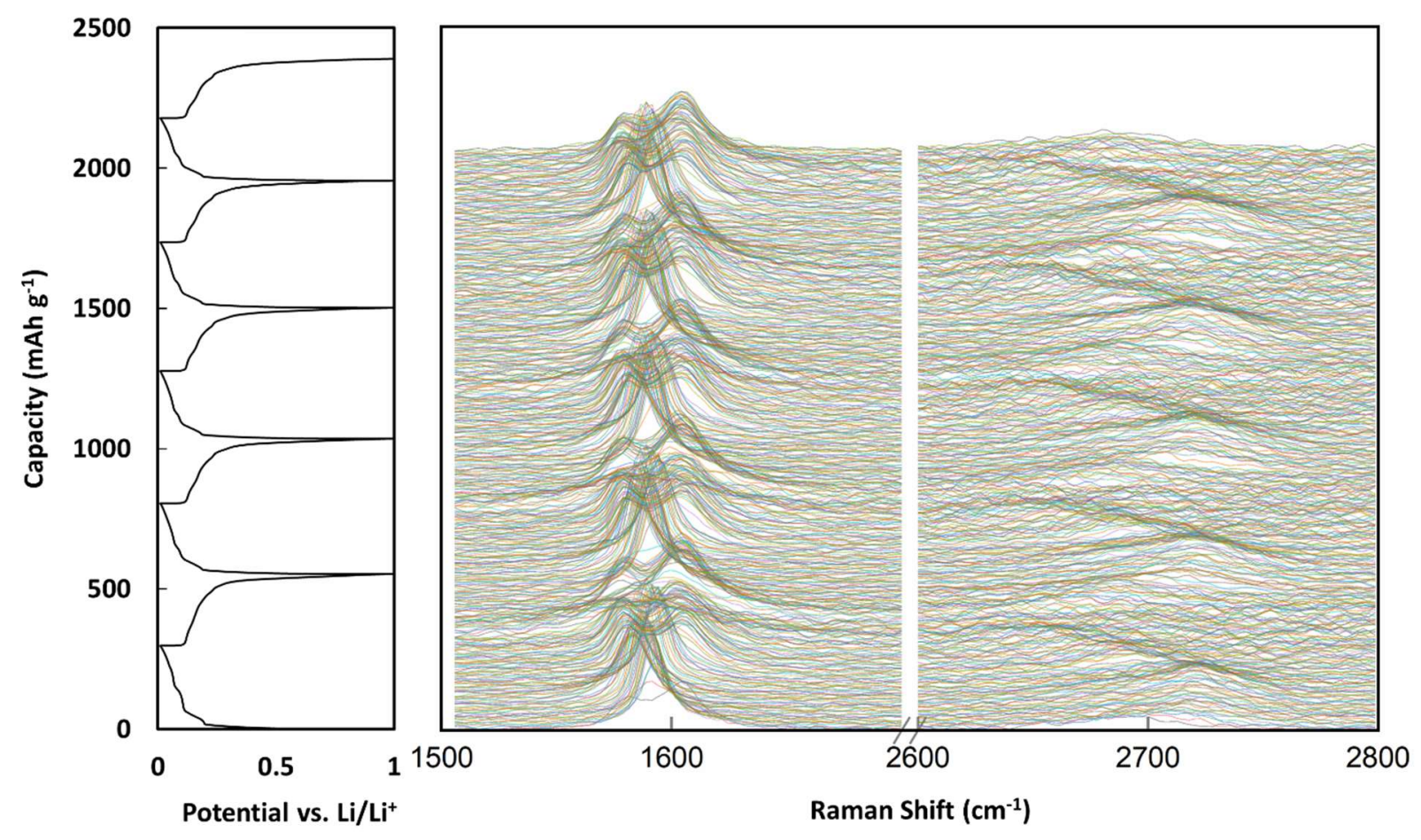

Figure 59: Five consecutive charge/discharge cycles as well as the corresponding operando Raman spectra of a graphite electrode in a half-cell vs. Li electrode performed under $\mathrm{C} / 2$. Graphite electrode exhibited reversible charge/discharge cycles, while $G$ and 2D band features in Raman spectra displayed a uniform transformation in each cycle. This experiment confirms that the Raman measurements do not interfere with the electrochemistry of the cell. 


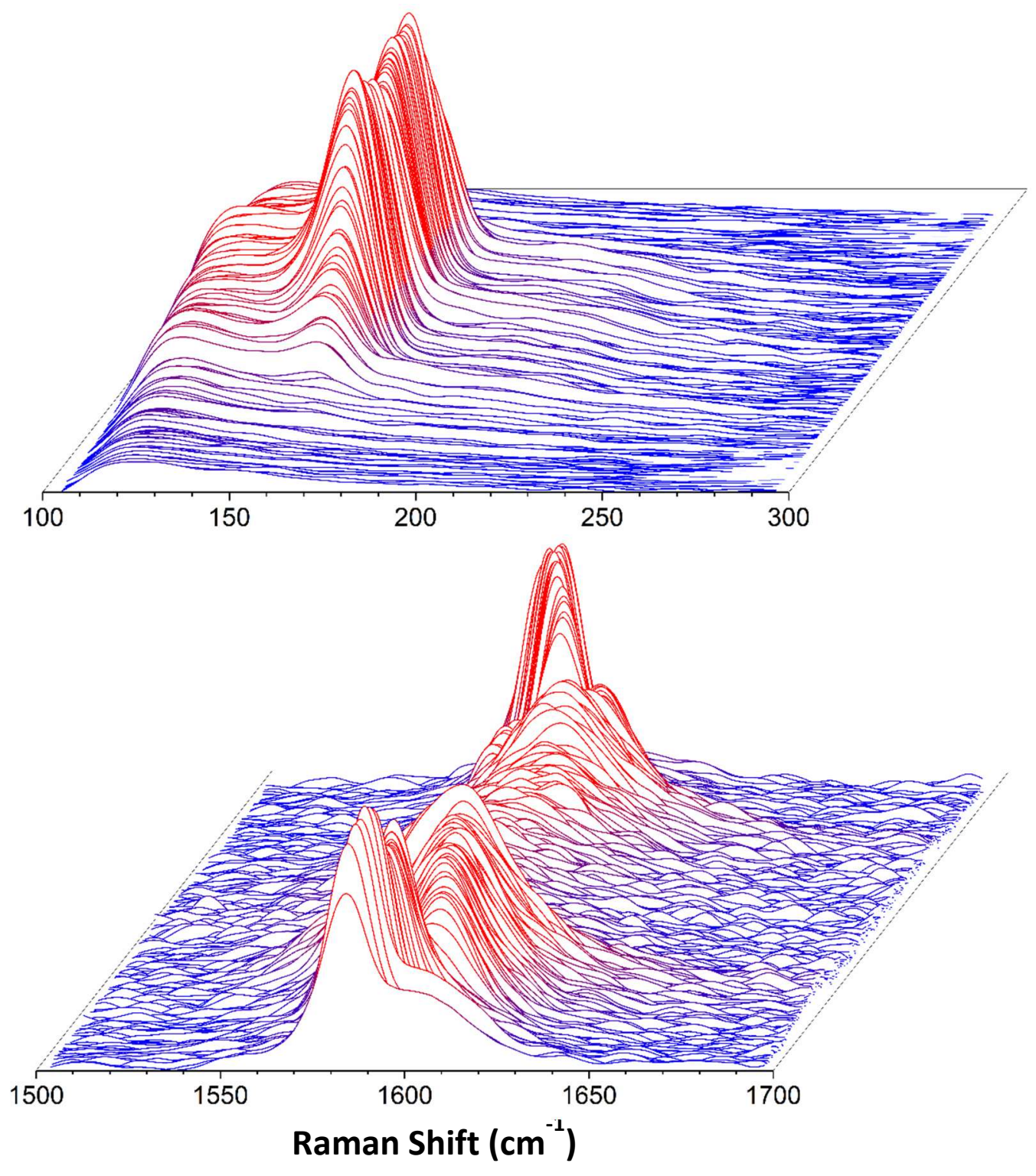

Figure S10: Operando Raman spectra including the low-frequency range obtained during a charge/discharge cycle of graphite electrode in a half-cell vs. Li electrode under C/20 rate. 


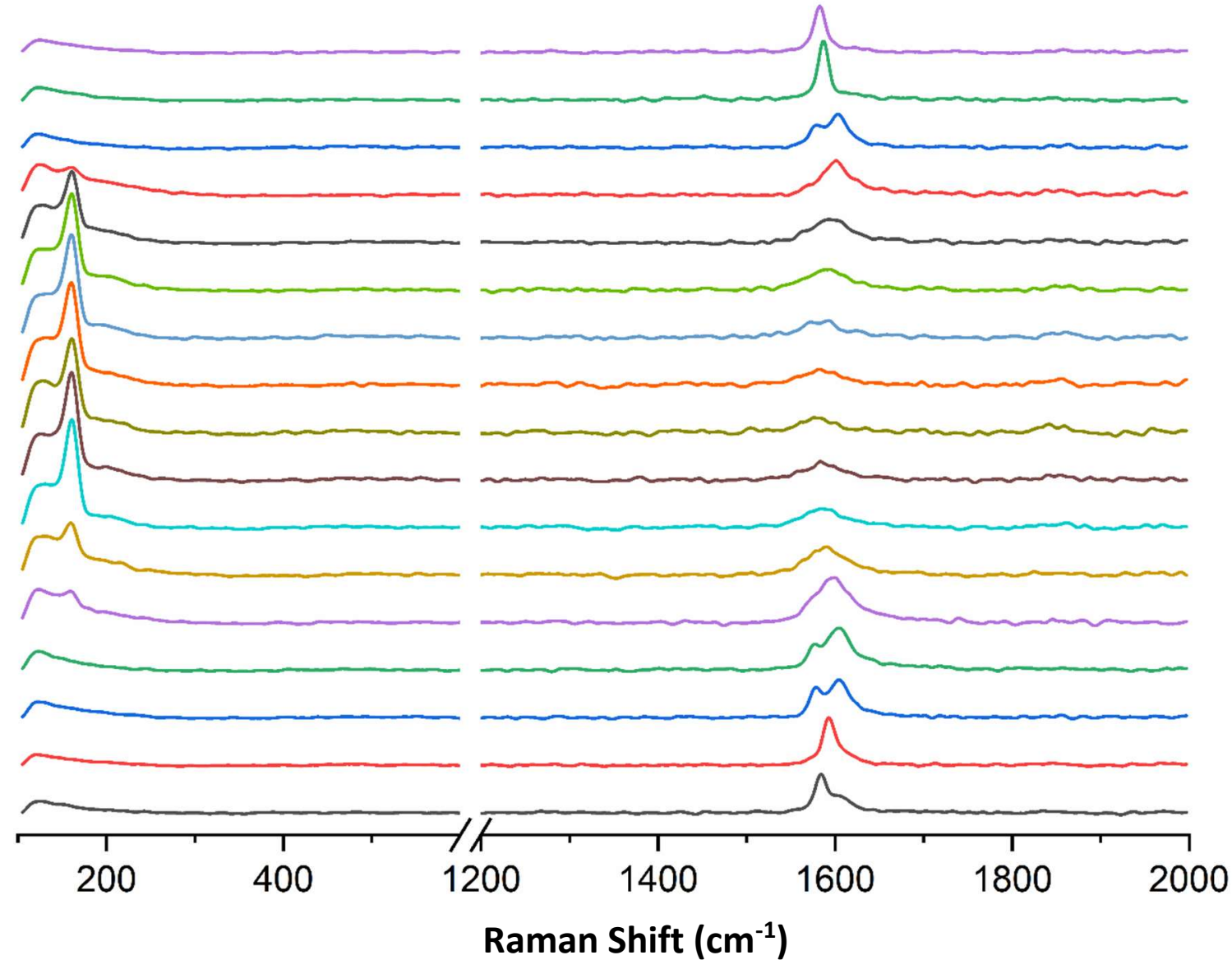

Figure S11: Operando Raman spectra recorded with 140 min intervals obtained during a charge/discharge cycle of graphite electrode in a half-cell vs. Li electrode under C/20 rate. 

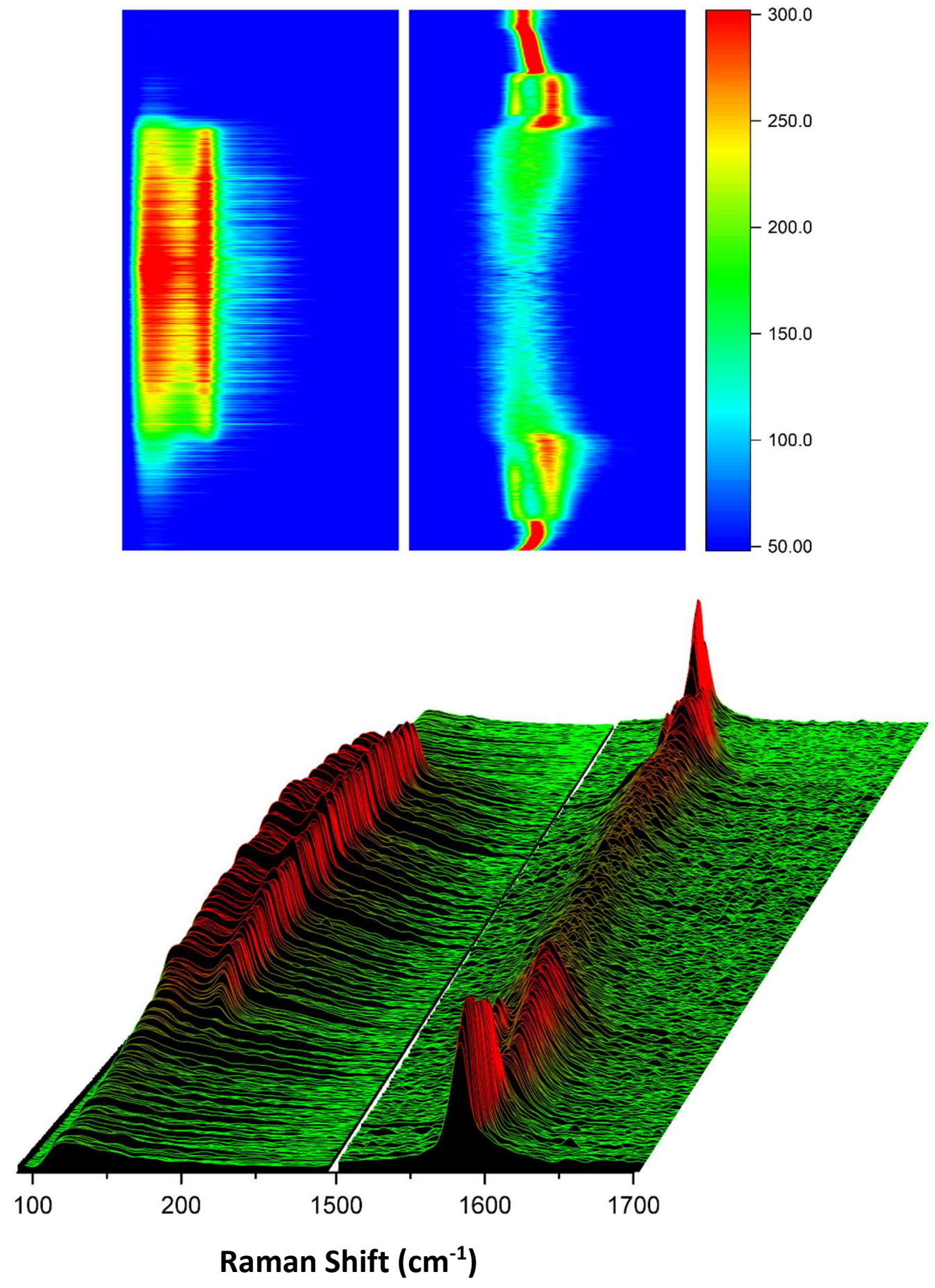

Figure S12: Operando Raman spectra as well as the corresponding contour plot including the lowfrequency range obtained from a typical charge/discharge cycle of graphite electrode in a halfcell vs. Li electrode performed under C/10. 

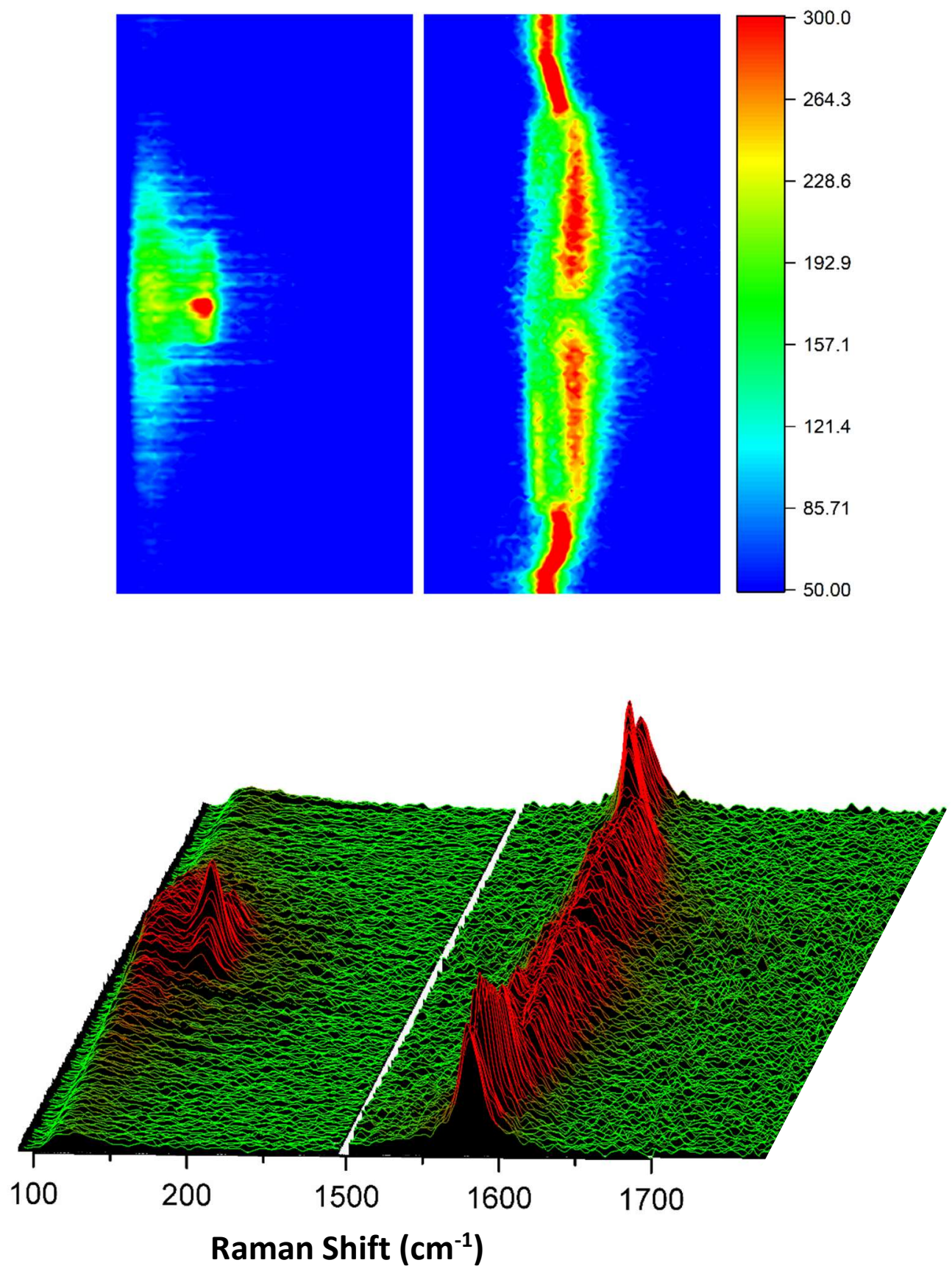

Figure S13: Operando Raman spectra as well as the corresponding contour plot including the lowfrequency range obtained from a typical charge/discharge cycle of graphite electrode in a halfcell vs. Li electrode performed under $\mathrm{C} / 2$. 


\section{$\mathrm{LiC}_{18}$}

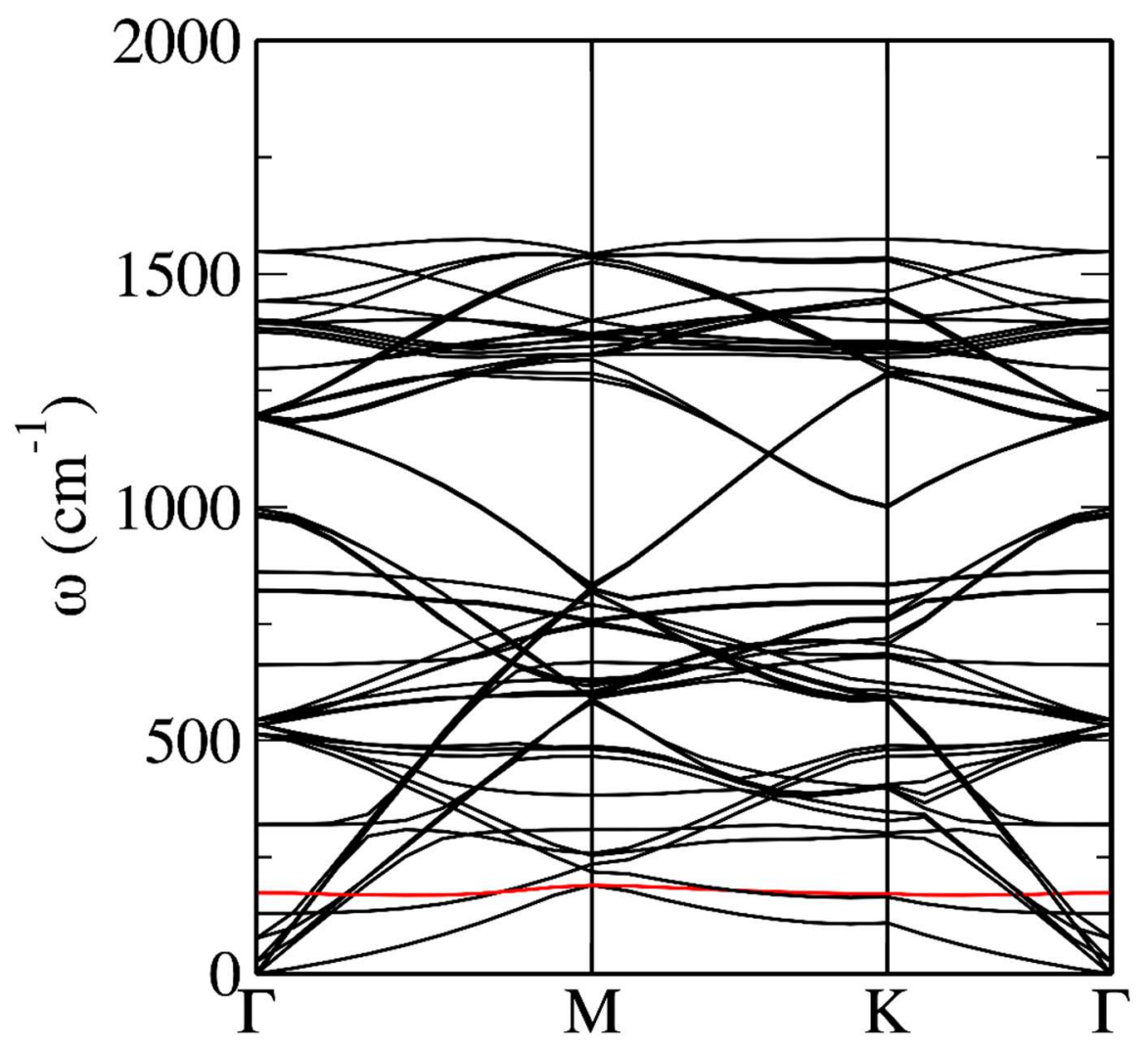

Figure S14: Phonon dispersion plot calculated for $\mathrm{LiC}_{18}$ using density functional theory. The lowfrequency Raman active mode is shown in red. 


\section{$\mathrm{LiC}_{12}$}

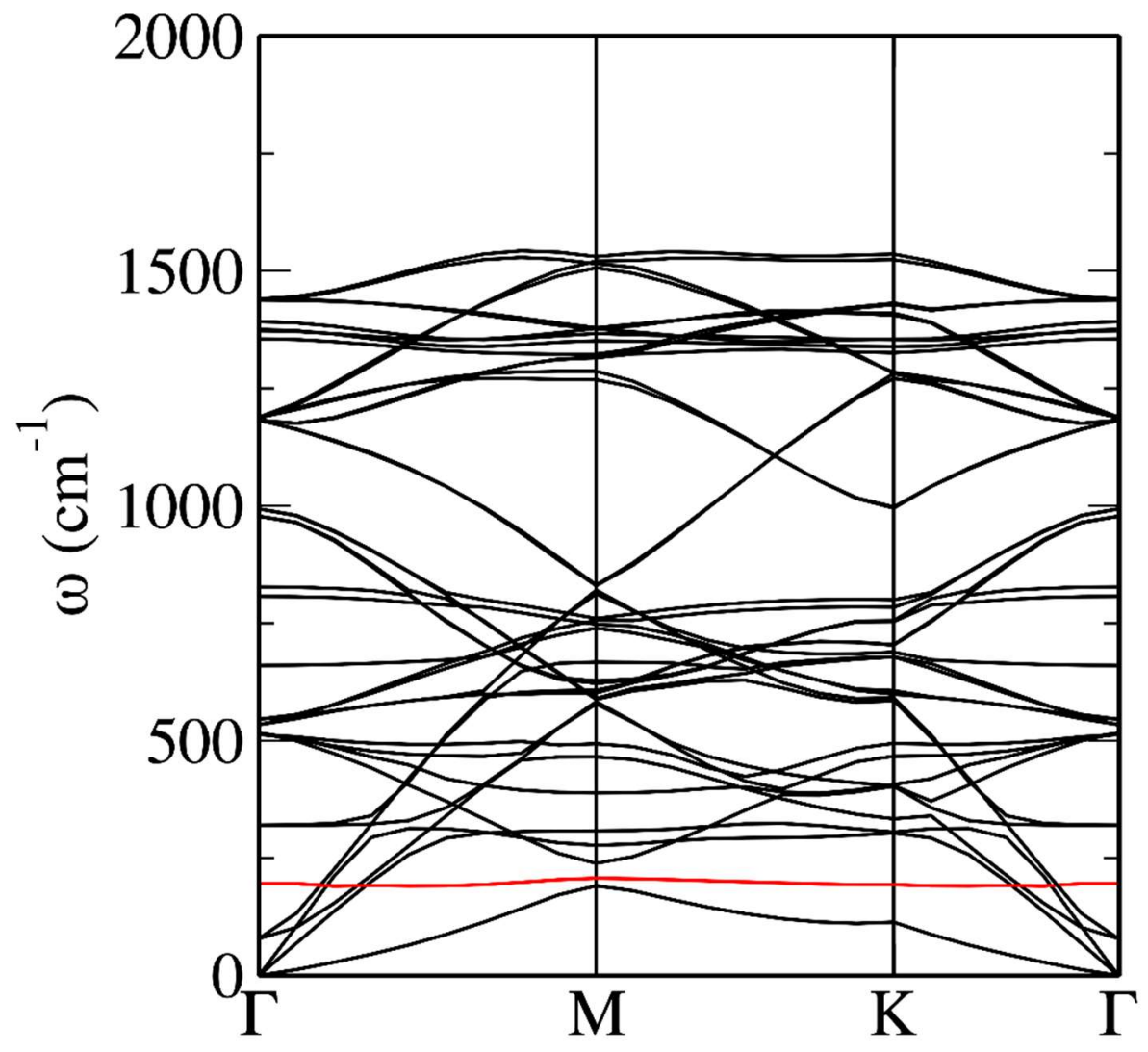

Figure S15: Phonon dispersion plot calculated for $\mathrm{LiC}_{12}$ using density functional theory. The lowfrequency Raman active mode is shown in red. 


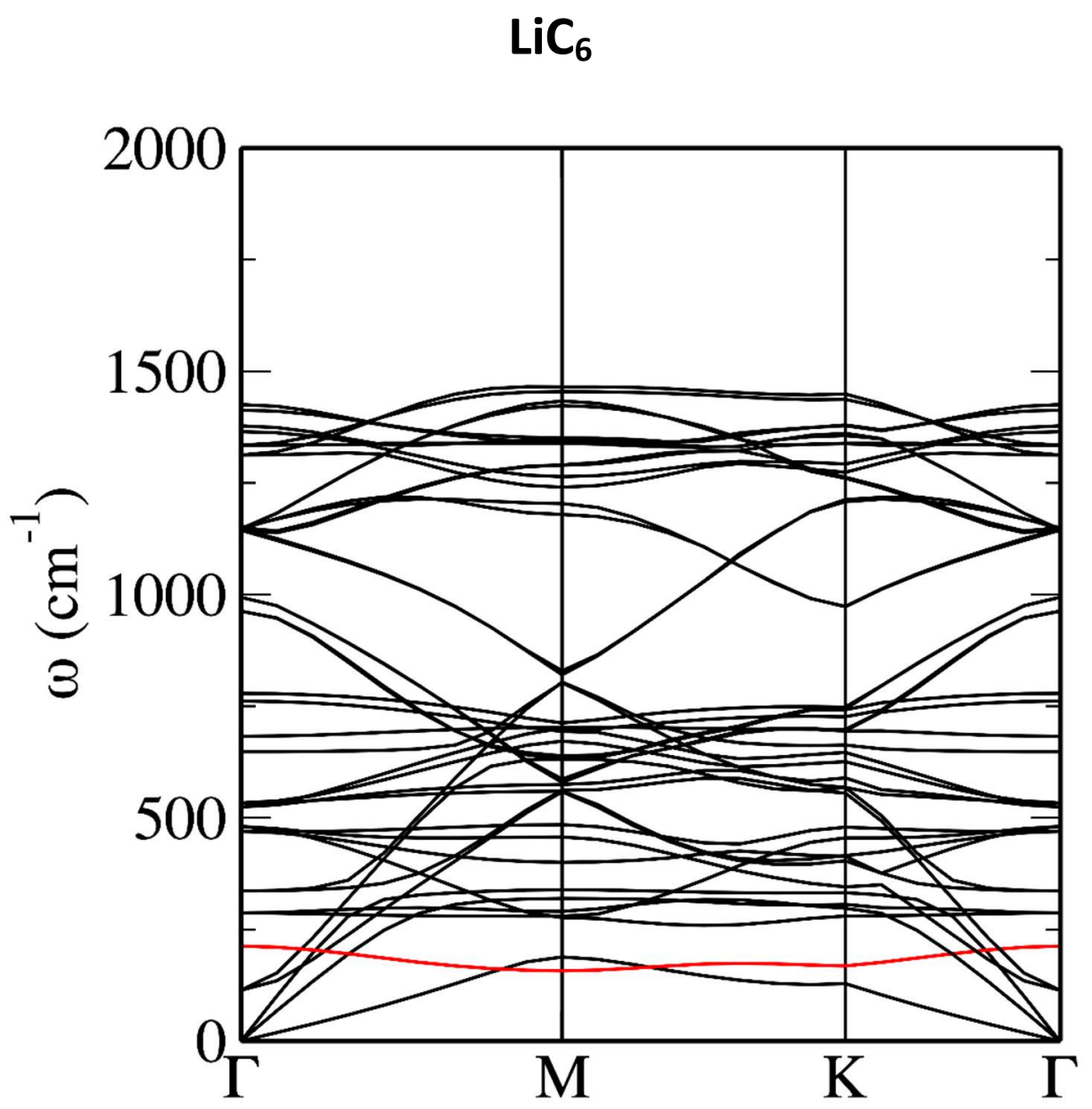

Figure S16: Phonon dispersion plot calculated for $\mathrm{LiC}_{6}$ using density functional theory. The lowfrequency Raman active mode is shown in red. 


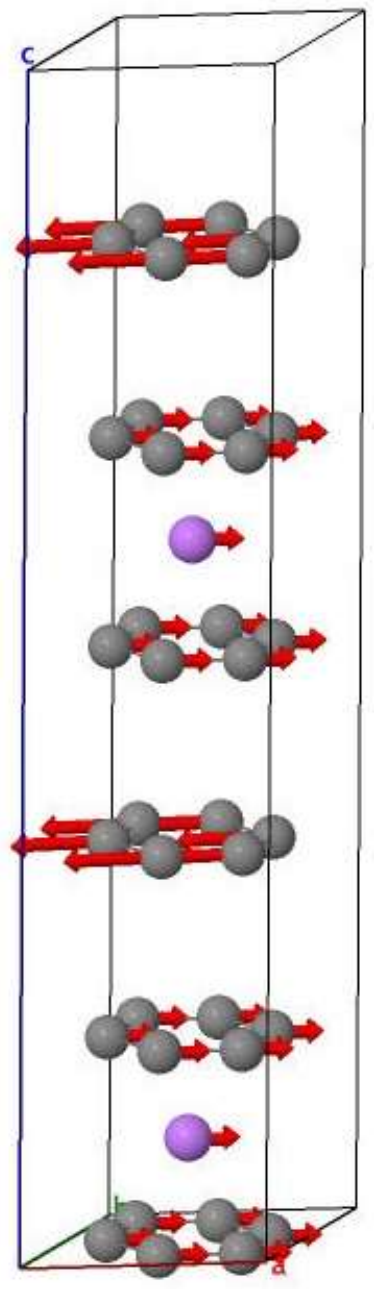

Figure S17: The displacement pattern of the Raman active mode calculated for $\mathrm{LiC}_{18}$ stacked in $A A$ sequence.

DFT calculations for $\mathrm{LiC}_{18}$ stacked in $A A$ sequence resulted in negative frequencies at the $\Gamma$-point. The displacement pattern of the negative frequencies involves displacing the first two layers of graphene and $\mathrm{Li}$ atom in one direction and displacing the third layer of graphene in the opposite direction, which implies $A A B$ stacking is more favorable. 


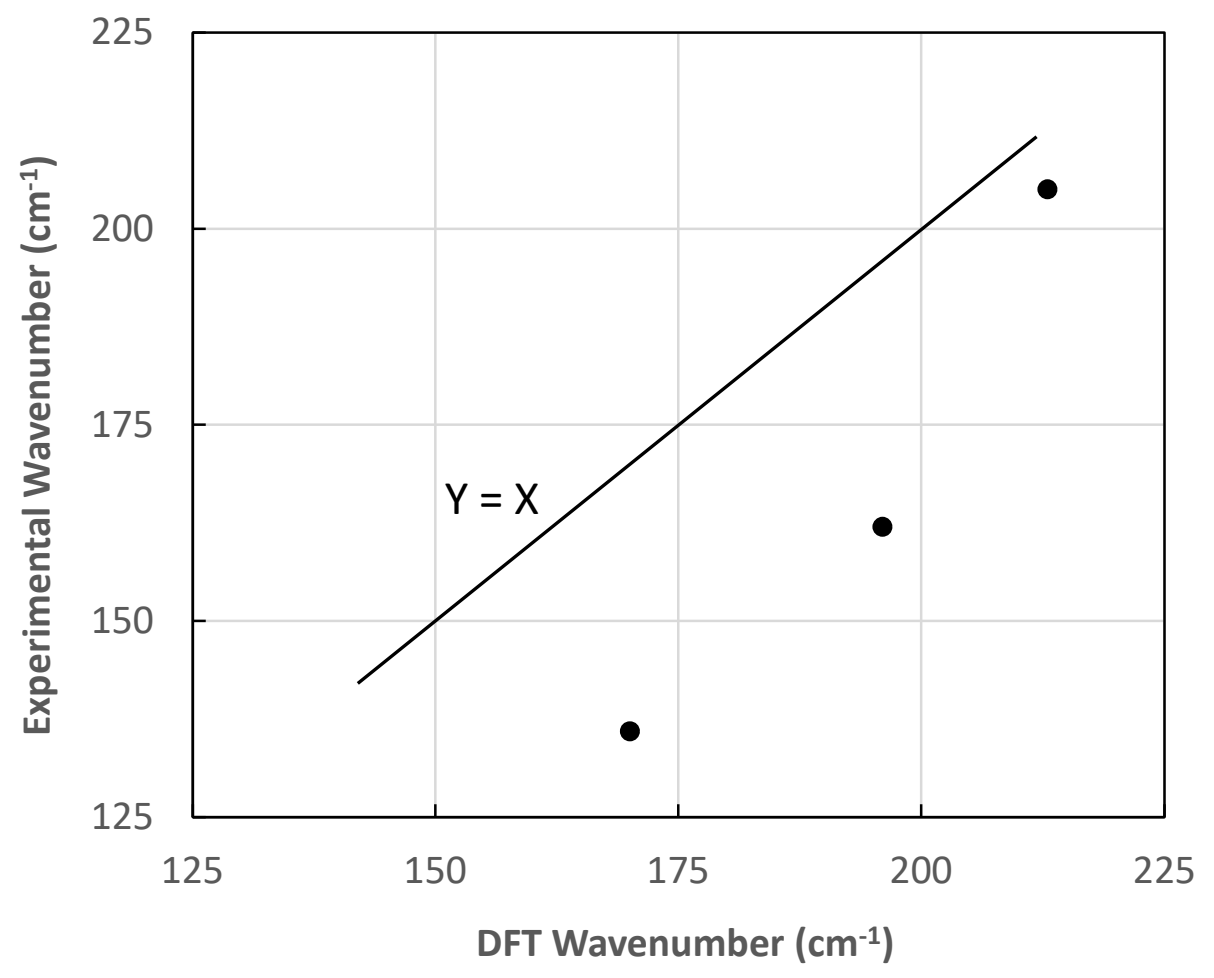

Figure S18: LMB frequencies obtained through ORS are plotted against those obtained via DFT calculations. 


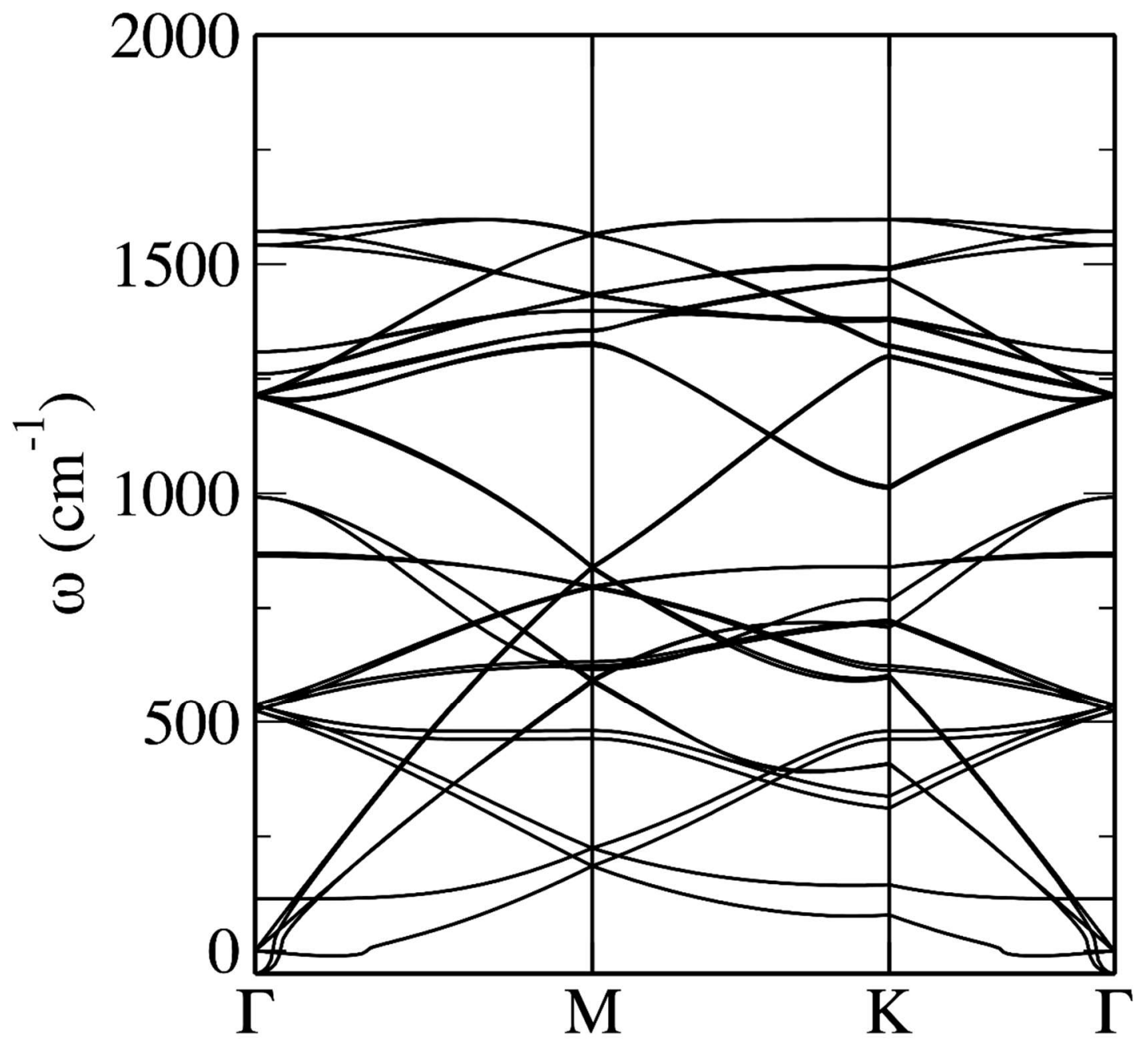

Figure S19: Phonon dispersion plot calculated for $C_{12}$ stacked in $A A$ sequence using density functional theory. 


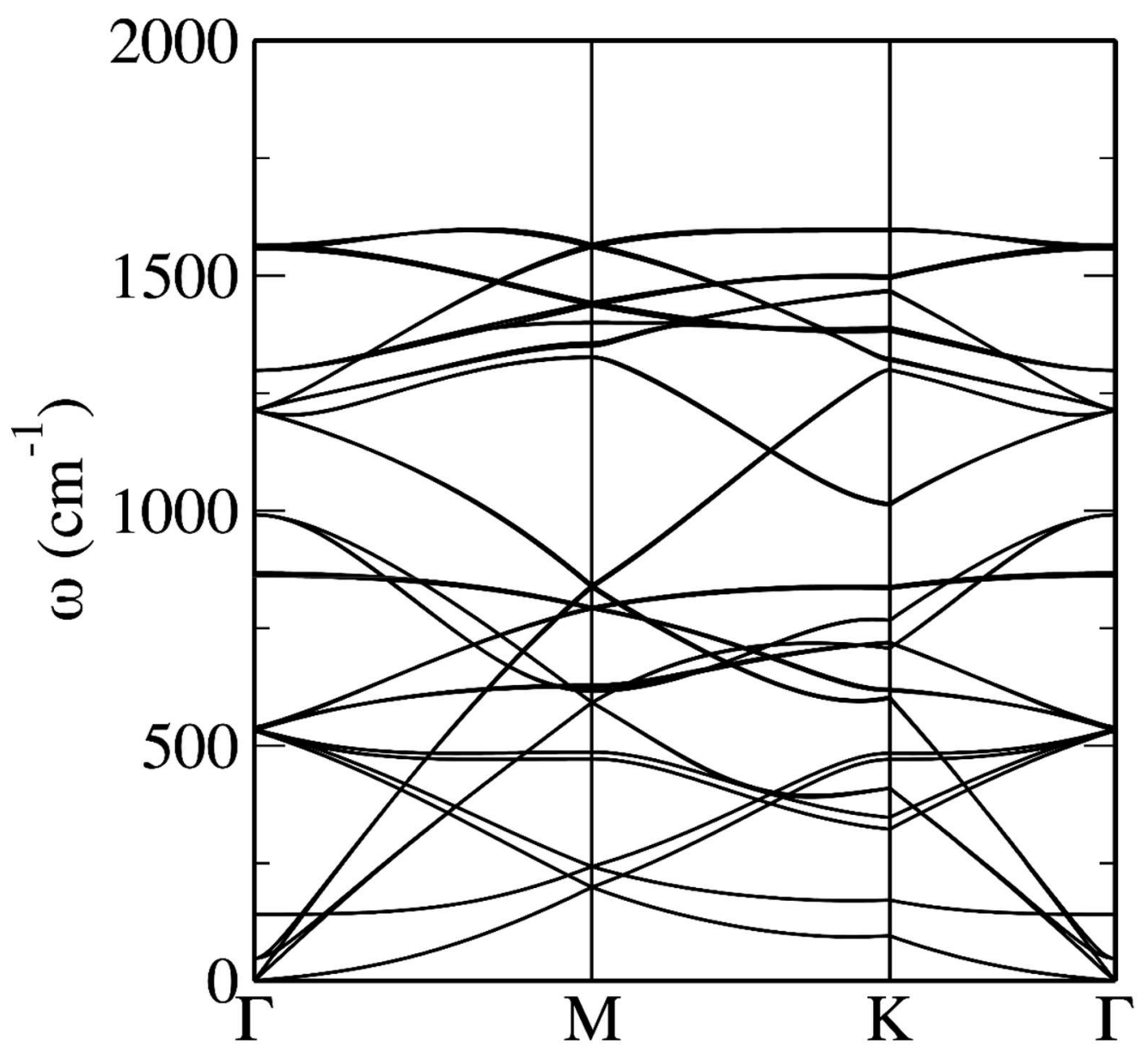

Figure S20: Phonon dispersion plot calculated for $C_{12}$ stacked in $A B$ sequence using density functional theory. 


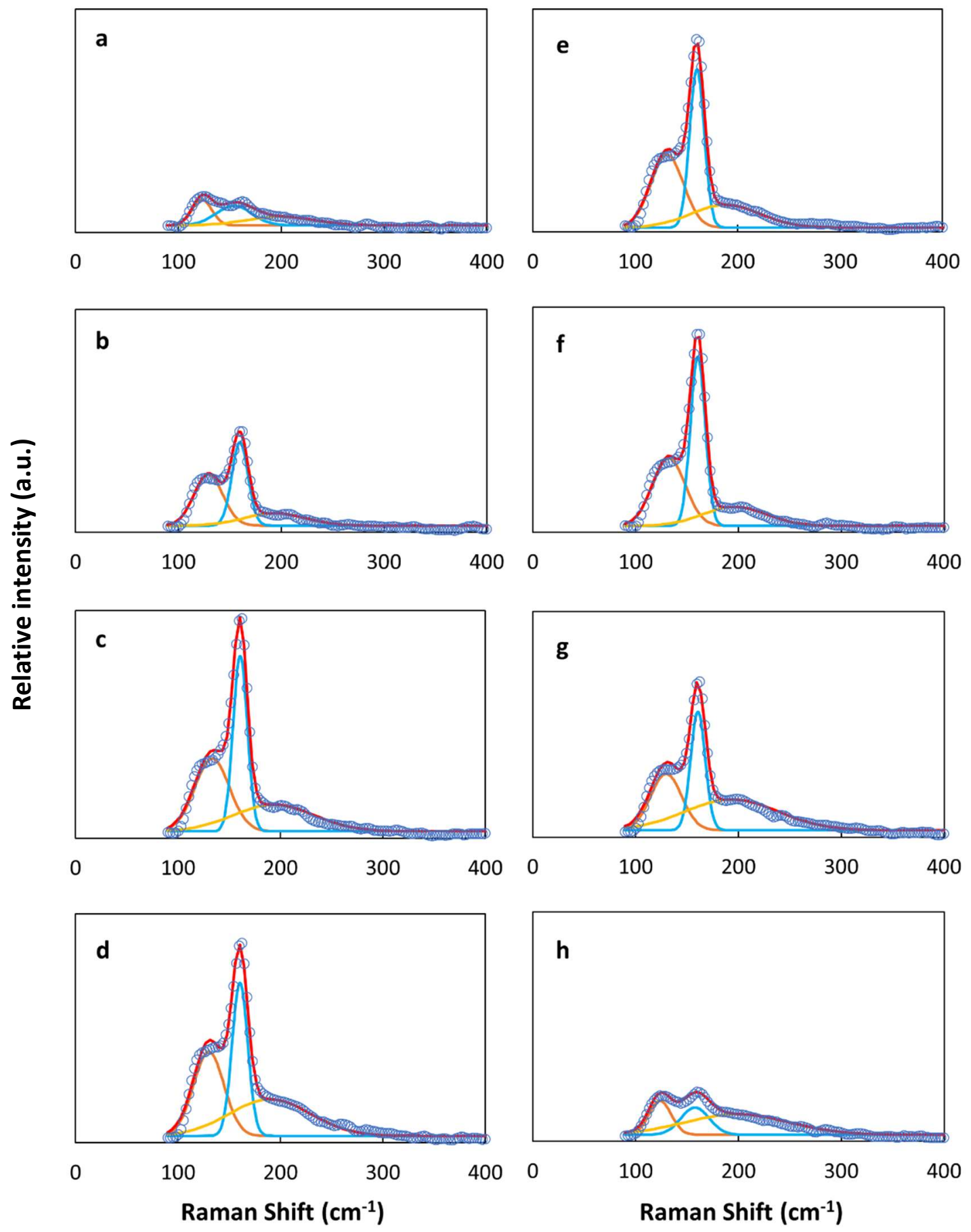

Figure S21: The deconvoluted experimental spectra at different points during stage 2-1 of Li intercalation and deintercalation under $\mathrm{C} / 20$. The spectra could be reasonably fitted with three Lorentzian peaks. 


\section{References:}

1. Clark, S. J.; Segall, M. D.; Pickard, C. J.; Hasnip, P. J.; Probert, M. J.; Refson, K.; Payne, M. C., First principles methods using CASTEP. Zeitschrift Fur Kristallographie 2005, 220 (56), 567-570.

2. Perdew, J. P.; Burke, K.; Ernzerhof, M., Generalized gradient approximation made simple. Phys. Rev. Lett. 1996, 77 (18), 3865-3868.

3. Grimme, S., Semiempirical GGA-type density functional constructed with a long-range dispersion correction. J. Comput. Chem. 2006, 27 (15), 1787-1799.

4. McNellis, E. R.; Meyer, J.; Reuter, K., Azobenzene at coinage metal surfaces: Role of dispersive van der Waals interactions. Physical Review B 2009, 80 (20), 205414.

5. Head, J. D.; Zerner, M. C., A Broyden-Fletcher-Goldfarb-Shanno Optimization Procedure for Molecular Geometries. Chem. Phys. Lett. 1985, 122 (3), 264-270. 